\section{Obsessions and compulsions in Asperger syndrome} and high-functioning autism

\author{
AILSA J. RUSSELL, DAVID MATAIX-COLS, MARTIN ANSON \\ and DECLAN G. M. MURPHY
}

\begin{abstract}
Background Obsessive-compulsive behaviours are common and disabling in autistic-spectrum disorders (ASD) but little is known about how they compare with those experienced by people with obsessive-compulsive disorder (OCD).
\end{abstract}

Aim To make such a comparison.

Method A group of adults with highfunctioning ASD $(n=40)$ were administered the Yale-Brown ObsessiveCompulsive Scale and Symptom Checklist and their symptoms compared with a gender-matched group of adults with a primary diagnosis of OCD $(n=45)$. OCD symptoms were carefully distinguished from stereotypic behaviours and interests usually displayed by those with ASD.

\begin{abstract}
Results The two groups had similar frequencies of obsessive-compulsive symptoms, with only somatic obsessions and repeating rituals being more common in the OCD group. The OCD group had higher obsessive-compulsive symptom severity ratings but up to $50 \%$ of the ASD group reported at least moderate levels of interference from their symptoms.
\end{abstract}

Conclusions Obsessions and compulsions are both common in adults with high-functioning ASD and are associated with significant levels of distress.

Declaration of interest None.
Obsessional/repetitive behaviours are a core feature of autistic-spectrum disorders (ASD; comprising autism and Asperger syndrome). These symptoms are disabling (Howlin, 1997) but little is known about how they compare with those experienced by people with obsessive-compulsive disorder (OCD). In the only previous study addressing this question, McDougle et al (1995) administered the Yale-Brown Obsessive-Compulsive Scale and Symptom Checklist to 50 individuals with autism and 50 matched individuals with OCD. Those with autism were less likely to report obsessional thoughts or cleaning, checking and counting symptoms, but were more likely to report repeating, hoarding, touching, tapping and self-damaging behaviours than the participants with OCD. McDougle et al (1995) concluded that the repetitive thoughts and behaviours characteristic of autism differed significantly from those of OCD. However, since $50 \%$ of the autistic sample had below-average IQ and/or were mute, it remained unclear whether these patients had other symptoms (i.e. obsessions) that they were unable to report. We compared the frequency and severity of repetitive 'obsessional' thoughts and 'compulsive' behaviours of a group of highfunctioning adults with ASD with those in adults with OCD. We hypothesised that individuals with high-functioning ASD would describe obsessions as well as compulsions but that these would be less severe than in OCD.

\section{METHOD}

\section{Participants and diagnosis}

Study participants were 40 adults with ASD and 45 gender-matched adults with OCD. Participants with comorbid psychosis and/ or substance misuse, and measured IQ $<70$ were excluded. The local ethics committee approved the study and all participants signed a consent form.
Consecutive referrals to specialist autism services at the Maudsley Hospital in south London were approached about participating in the study. A diagnosis of ASD was determined using ICD-10 criteria (World Health Organization, 1992) for autism and Asperger syndrome. In addition, where parents were available (23 cases, $58 \%$ ) the Autism Diagnostic Interview (ADI; Le Couteur et al, 1989) was conducted. Of the adults with ASD, 36 (90\%) were diagnosed with Asperger syndrome and $4(10 \%)$ with high-functioning autism, with significant delay in the acquisition of spoken language delineating the latter according to ADI criteria. Each individual was subject to careful diagnostic assessment by a consultant psychiatrist specialising in ASD, and ICD-10 comorbid diagnoses were recorded in the admission and/or discharge medical reports. Mean Verbal and Performance IQs on the Wechsler Adult Intelligence Scale - third edition (WAIS-III; Wechsler, 1981) for the ASD group were 99.18 (s.d.=18.37) and 93.68 (s.d.=13.13), respectively. Participants with OCD were recruited from a specialist clinic in west London. All were diagnosed using semi-structured interviews by experienced clinicians using DSM-IV (American Psychiatric Association, 1994) or ICD-10 criteria.

\section{Severity and types of OCD symptoms}

The severity and types of obsessivecompulsive symptoms were measured using, respectively, the ten-item YaleBrown Obsessive-Compulsive Scale (Y-BOCS) (Goodman et al, 1989) and its ancillary Symptom Checklist (Y-BOCS-SC). The Y-BOCS-SC is a list of more than 50 examples of obsessions and compulsions grouped thematically into seven main groups of obsessions and six of compulsions. A score of 0 (absent symptom) or 1 (present symptom) was given to each of the 13 main types of obsessions and compulsions. Miscellaneous obsessions and compulsions were not included in the analyses, as they contain many different types of symptoms that do not naturally cluster together.

For the purpose of this study it was important that only genuine obsessions and compulsions were recorded in the Y-BOCS-SC, and an effort was made to distinguish those from the stereotypic behaviours and interests usually displayed 
by people with ASD. At the beginning of the interview, each ASD participant was asked what was meant by an 'obsession'. Participants who offered a definition consistent with an obsessive-compulsive symptom were prompted to report any similar experiences they had before the Y-BOCSSC was administered. If a participant defined an obsession as 'something one is very interested in', the examiner agreed that this could be so, but explained that the interview was about another type of obsession; a definition was then offered and the participant was again prompted to report their own experiences. If the participant did not have any obsessions or did not understand the question, an example of a typical obsessive-compulsive symptom was offered. This procedure was repeated for compulsions. Symptoms were only rated if they caused some degree of discomfort and interfered with the patient's daily life. Two raters independently scored the Y-BOCS-SC in a subsample of the ASD group $(n=4)$ and consensus reliability for the symptom checklist and the severity scale was established. Interrater agreement was calculated as an overall percentage of items where both observers agreed, divided by the total number of items. A high level of agreement was achieved.

\section{RESULTS}

\section{Demographic characteristics and comorbidity}

Of the 40 people with ASD, 34 (85\%) were male and $6(15 \%)$ were female. Similarly, $39(87 \%)$ of the OCD group were male and $6(13 \%)$ were female. People with OCD (mean age $=36.6$ years, s.d. $=11.5$ ) were significantly older than those with ASD (mean age $=27.9$ years, s.d. $=8.5$ ) $(t=3.96$, d.f. $=83, P<0.001)$.

Comorbid ICD-10 diagnoses in the ASD group were not available for 3 $(7.5 \%)$ patients. Seventeen $(42.5 \%)$ patients did not receive an additional psychiatric diagnosis. Of the $20(50 \%)$ participants with comorbid diagnoses, 11 $(27.5 \%)$ received a diagnosis of affective disorder, $10(25 \%)$ were diagnosed with comorbid OCD, $3(7.5 \%)$ had a discharge diagnosis of schizophrenia and $4(10 \%)$ had an anxiety disorder other than OCD.

\section{Types of obsessive-compulsive symptoms}

A similar frequency of obsessions and compulsions was observed across the two groups (Table 1). The OCD group did, however, report significantly higher frequencies of somatic obsessions and repeating and checking compulsions. A discriminant function analysis (stepwise procedure) showed that group membership was best predicted on the basis of a single type of obsession, somatic obsessions $(F=15.845$, d.f. $=1,83, P<0.0001)$, and a single type of compulsion, repeating compulsions $(F=10.460$, d.f. $=2,82, P<0.0001)$. Both were more frequent in the OCD group. None of the other symptoms improved the fit of the model or were retained in the analysis.

The analyses were repeated comparing the symptom types endorsed by the group with ASD plus a formal diagnosis of OCD $(n=10)$ with those endorsed by the OCD group. Somatic obsessions were significantly more frequent in the group with OCD alone $\left(\chi^{2}=6.19\right.$, d.f. $\left.=1, P=0.013\right)$, with $(10 \%)$ of patients in the ASD plus OCD group endorsing this symptom type compared with $24(53 \%)$ patients in the OCD group. Sexual obsessions were more commonly reported by patients in the ASD plus OCD group $\left(\chi^{2}=3.9\right.$, d.f. $=1$, $P=0.046)$, with $7(70 \%)$ of the group endorsing this symptom type compared with $16(36 \%)$ of the OCD group. A discriminant function analysis (stepwise procedure) showed that group membership was best predicted by somatic obsessions ( $F=6.730$, d.f. $=1,53, P<0.01)$ and sexual obsessions $(F=7.650$, d.f. $=2,52, P<0.001)$. No other variables were retained in the analysis.

\section{Number of endorsed symptoms}

The OCD group reported a mean of 10.4 (s.d.=7.4) obsessions and 7.8 (s.d.=4.54) compulsions compared with a mean of 6.7 (s.d.=5.36) obsessions $(t=2.609$, d.f. $=83$, $P=0.001$ ), and 4.4 (s.d. $=4.1$ ) compulsions $(t=3.561$, d.f. $=83, P=0.001)$ in the ASD group.

\section{Symptom severity}

Y-BOCS severity scores were available for 38 participants with ASD and 44 with OCD. As predicted, the OCD group scored significantly higher on the Y-BOCS total scales $(\mathrm{OCD}$ mean $=23.8$, s.d.=7.1, ASD mean $=16.2, \quad$ s.d. $=9.7 ; \quad t=4.1, \quad$ d.f. $=81$, $P<0.001$ ), obsessions (OCD mean $=11.6$, s.d.=3.9; ASD mean=8.46, s.d. $=6.1$; $t=2.8$, d.f. $=79, P=0.005)$ and compulsions (OCD mean $=12.09$, s.d. $=3.9 ; \quad$ ASD mean $=7.6, \quad$ s.d. $=5.3 ; \quad t=4.3, \quad$ d.f. $=80$, $P<0.0001$ ).

Breakdown of the Y-BOCS items in the ASD group revealed that $15(39 \%)$ rated the time taken by their obsessions to be 'moderate' (1-3 h/day) or above; 18 $(47 \%)$ reported at least moderate levels of interference and $23(60 \%)$ reported at least moderate levels of distress as a result of their obsessions. Compulsions occupied at least $1-3 \mathrm{~h} /$ day in $12(26 \%)$ of the group

Table I Participants with ASD $(n=40)$ and OCD $(n=45)$ reporting symptoms from the Yale-Brown Obsessive-Compulsive Symptom Checklist

\begin{tabular}{lcccc}
\hline Symptoms & $\begin{array}{c}\text { ASD group } \\
n(\%)\end{array}$ & $\begin{array}{c}\text { OCD group } \\
n(\%)\end{array}$ & $\begin{array}{c}\chi^{2} \\
(\text { d.f. }=I)\end{array}$ & $P$ \\
\hline Obsessions & & & & \\
Aggressive & $20(50.0)$ & $29(64.4)$ & 1.810 & 0.179 \\
Contamination & $24(60.0)$ & $31(68.9)$ & 0.733 & 0.392 \\
Sexual & $13(32.5)$ & $16(35.6)$ & 0.088 & 0.767 \\
Hoarding & $17(42.5)$ & $23(51.1)$ & 0.630 & 0.427 \\
Religious & $12(30.0)$ & $15(33.3)$ & 0.109 & 0.742 \\
Symmetry & $22(55.0)$ & $27(60.0)$ & 0.217 & 0.641 \\
Somatic & $6(15.0)$ & $24(53.3)$ & 13.626 & $<0.001$ \\
Compulsions & & & & \\
Cleaning & $22(55.0)$ & $30(66.7)$ & 1.214 & 0.271 \\
Checking & $24(60.0)$ & $37(82.2)$ & 5.161 & 0.023 \\
Repeating & $17(42.5)$ & $31(68.9)$ & 5.999 & 0.014 \\
Counting & $4(10.0)$ & $11(24.4)$ & 3.040 & 0.080 \\
Arranging & $10(25.0)$ & $15(33.3)$ & 0.708 & 0.400 \\
Hoarding & $12(30.0)$ & $19(42.2)$ & 1.365 & 0.243 \\
\hline
\end{tabular}

ASD, autistic-spectrum disorder; OCD, obsessive-compulsive disorder. 
with ASD; these led to moderate or above levels of interference in 16 (42\%), and 21 $(56 \%)$ reported at least moderate levels of anxiety if prevented from performing their rituals.

\section{DISCUSSION}

Adults with ASD and average IQ reported a high frequency of both obsessions and compulsions as defined in standard nomenclatures, i.e. being experienced as intrusive, distressing and time-consuming. Only somatic obsessions, repeating and checking rituals were significantly more frequent in a gender-matched OCD group. A quarter of the ASD group also met formal ICD-10 diagnostic criteria for OCD. When this ASD plus OCD group was compared with the OCD group for symptom type, somatic obsessions were reported significantly more frequently in the OCD group and sexual obsessions more frequently in the ASD plus OCD group.

In the only previous study investigating obsessive and compulsive symptoms in ASD (McDougle et al, 1995), particpants with ASD and OCD were distinguished by seven types of repetitive thoughts and behaviours, with the latter being predominant. The authors postulated that this could be accounted for by the below average general IQ and verbal abilities of the ASD group; our findings support this.

It is intriguing that somatic obsessions were much more frequent in the OCD group than in the ASD group and even in the ASD plus OCD group. There is minimal literature on the identification and reporting of physical health problems in ASD. However, it is a common clinical observation and families often report that these individuals have difficulties reporting any physical complaints; instead increased agitation/behavioural disturbance and distress may be seen. In the Autism Diagnostic Interview - Revised (ADI-R; Lord et al, 1994), lack of reporting and seeking comfort following physical illness and injury is an important diagnostic feature of a pervasive developmental disorder during childhood. Lack of focus on internal physiological discomfort would appear to directly translate into a lack of focus for anxiety in this group.

\section{Implications}

These results may have important implications for both clinical practice and

\section{CLINICAL IMPLICATIONS}

Genuine obsessions and compulsions are more frequent and distressing in autisticspectrum spectrum disorders (ASD) than previously thought.

- Obsessive-compulsive disorder (OCD) symptoms should be carefully distinguished from other common repetitive behaviours and special interests in ASD.

- Patients with prominent obsessive-compulsive symptoms may benefit from standard treatments for OCD but controlled trials are needed to confirm this.

\section{LIMITATIONS}

- The study lacked structured diagnostic interviews. However, all patients were carefully diagnosed by experienced psychiatrists.

- The OCD sample was significantly older than the ASD group and it is unclear if this had an effect on the results.

- Participants in the ASD group were recruited from a specialist clinic that treats patients with high levels of comorbidity. The results may not be applicable to other ASD clinics.

AILSA J. RUSSELL, MSc, Department of Psychology, DAVID MATAIX-COLS, PhD, Department of Psychology and Department of Psychological Medicine, MARTIN ANSON, MSc, Department of Psychology, DECLAN G. M. MURPHY, MD, Department of Psychological Medicine, Institute of Psychiatry, King's College London, UK

Correspondence: Ms Ailsa J. Russell, Department of Psychology, Institute of Psychiatry, PO Box 77, De Crespigny Park, Denmark Hill, London SE5 8AF. Tel: 0207848 0655; fax: 0207848 5006; e-mail: a.russell@iop.kcl.ac.uk

(First received 24 February 2004, final revision 14 September 2004, accepted 30 October 2004)

research. From a therapeutic perspective, clinicians need to consider the possibility of significant obsessive-compulsive symptoms in ASD, rather than classifying repetitive phenomenology as characteristic of ASD. Proper assessment to distinguish these two types of phenomena is important.

Additionally, individuals with ASD who have distressing levels of obsessivecompulsive symptoms may benefit from standard treatments for OCD such as serotonin reuptake inhibitors (McDougle et al, 2000) and cognitive and behavioural therapies (Marks, 2003), but controlled trials are needed to evaluate the efficacy of these interventions in ASD. Executive function deficits and a preference for local rather than global information processing have been reported in both ASD and OCD (Ozonoff et al, 1991; Savage et al, 1999). However, it is not known whether obsessive-compulsive behaviours in OCD and ASD share a common neural basis. We hope that the current results will stimulate research in these areas.

Obsessional thoughts are not in themselves uncommon and have been reported by $80 \%$ of the general population (Rachman \& De Silva, 1978). The cognitive style and deficits that have been empirically associated with ASD (Happe, 1994) may influence the way in which these 'normal' phenomena are appraised. High levels of general anxiety are typical in ASD, and this may also modulate the evolution of obsessive-compulsive symptoms.

\section{REFERENCES}

American Psychiatric Association (1994) Diagnostic and Statistical Manual of Mental Disorders (4th edn) (DSM-IV). Washington, DC: APA.

Goodman, W. K., Price, L. H., Rasmussen, S. A., et al (1989) The Yale-Brown Obsessive Compulsive Scale I: development, use and reliability. Archives of General Psychiatry, 46, 1006-10II. 
Happe, F. G. E. (1994) Annotation: current psychological theories of autism: the "theory of mind" account and rival theories. Journal of Child Psychology and Psychiatry, 35, 215-229.

Howlin, P. (1997) Autism: Preparing for Adulthood. London: Routledge.

Le Couteur, A., Rutter, M., Lord, C., et al (1989) Autism Diagnostic Interview: A semi-structured interview for parents and caregivers of autistic persons. Journal of Autism and Developmental Disorders, 19 363-387.

Lord, C., Rutter, M. \& Le Couteur, A. (1994) Autism Diagnostic Interview - Revised: a revised version of a diagnostic interview for caregivers of individuals with possible pervasive developmental disorders. Journal of Autism and Developmental Disorders, 24, 659-685.
Marks, M. (2003) Cognitive therapy for obsessive compulsive disorder. In Obsessive Compulsive Disorder: Theory, Research and Treatment (eds R. G. Menzies \& P. De Silva), pp. 275-290. Chichester: Wiley.

McDougle, C. J., Kresch, L. E., Goodman, W. K., et a (1995) A case-controlled study of repetitive thoughts and behaviour in adults with autistic disorder and obsessive-compulsive disorder. American Journal of Psychiatry, I52, 772-777.

\section{McDougle, C. J., Kresch, L. E., Posey, D. J. (2000)}

Repetitive thoughts and behavior in pervasive developmental disorders: treatment with serotonin reuptake inhibitors. Journal of Autism and Developmental Disorders, 30, 427-435.

Ozonoff, S., Pennington, B. F. \& Rogers, S. J. (1991) Executive function deficits in high-functioning autistic individuals: relationship to theory of mind. Journal of Child Psychology and Psychiatry, 32, II07-1122.

Rachman, S. \& De Silva, P. (1978) Abnormal and normal obsessions. Behaviour Research and Therapy, 16 233-238.

Savage, C. R., Baer, L. \& Keuthen, N. J. (1999)

Organizational strategies mediate nonverbal memory impairment in obsessive-compulsive disorder. Biological Psychiatry, 45, 905-916.

Wechsler, D. (198I) Manual for the Wechsler Adult Intelligence Scales - Revised. San Antonio, TX Psychological Corporation.

World Health Organization (1992) The ICD-10 Classification of Mental and Behavioral Disorders. Geneva: WHO. 